compression depth ranges (peak $45.6 \mathrm{~mm}$ ). Optimal CPR efforts may be achievable in suspected drowning victims while wearing a PFD, but did not reach current AHA compression depth guidelines. While there was a statistically significant difference in compression depth achievable among the 3 varieties of PFDs, its clinical significance (3-4 mm in depth) is uncertain. Further studies are needed to test other PFDs, in particular those that are used in the commercial rafting industry.

\section{Low-Cost, Highly Adaptable Mannequin for Use in Wilderness Medicine Simulation}

Joshua Nichols; Stephanie Lareau

Virginia Tech Carilion Clinic, Roanoke, VA, USA

Introduction.-Simulation-based learning is an effective means of teaching disease management and procedural skills for treating critically ill patients in the austere environment. Although high fidelity mannequins can simulate multiple disease states and procedures, it is usually cost prohibitive to use such expensive equipment in the wilderness. It is therefore common practice for simulations to involve a "live actor" patient combined with standalone anatomical models for conducting procedures. This arrangement has certain limitations, including putting the live actor at risk as well as inability to simulate certain disease states. We seek to understand the feasibility of creating a low-cost mannequin platform that can be adapted to simulate various disease states and procedural skills.

Objective.-To determine the feasibility of creating a simulation mannequin for less than $\$ 500$ that can simulate heart and lung sounds, cricothyrotomy, tension pneumothorax needle decompression, and arterial injury.

Methods.-A polystyrene adult male mannequin (\$115) was purchased from AMT Mannequins. This mannequin then was modified to allow for simulated procedures. A Bluetooth speaker $(\$ 20)$ was mounted in the chest that could play heart and lung sounds. The anterior neck was removed and a cricothyrotomy simulator was constructed in its place using foam tape, gauze pads, ketchup packets, and ventilator tubing. A tension pneumothorax needle decompression simulator was placed in the right midclavicular line using foam tape, gauze, and InstaMorph moldable plastic (\$35), which was used to construct custom ribs. A femoral arterial injury was simulated using a $1 \mathrm{~L}$ bag of normal saline in a pressure bag mounted inside the torso, connected to IV tubing running into the thigh.

Results. - The total cost of the custom mannequin was approximately $\$ 170$.

Conclusions.- It is feasible to construct an inexpensive simulation mannequin that can be used to simulate heart and lung sounds and for practicing multiple procedural skills.

\section{Which Matters More for Backcountry Preparedness: Wilderness or Medical Training?}

Michael D. Yue ${ }^{1}$; David W. Spivey ${ }^{1}$; Daniel B. Gingold ${ }^{1}$; Douglas G. Sward ${ }^{1,2}$

${ }^{1}$ Department of Emergency Medicine, University of Maryland School of Medicine, Baltimore, MD, USA, ${ }^{2}$ Program in Trauma, University of Maryland School of Medicine, Baltimore, MD, USA

Objective.-To identify how previous medical and wilderness training correlate to levels of preparedness for acute mountain sickness (AMS) as well as illness and injury in backcountry hikers. Few previous studies have directly assessed preparedness in the backcountry.

Methods.-We conducted a cross-sectional, convenience survey on trails in 4 sections of the Rocky Mountain National Park during 2 weeks in July and August 2015. The study group consisted of 379 hikers in the backcountry who consented to a written survey that assessed demographics, wilderness experience, altitude experience, hiking equipment, communications devices, and pretrip planning

Results. - Factors including wilderness training (WFA/WFR/WEMT), wilderness experience, and altitude experience all affected hiker preparedness. Respondents with medical training were more prepared for injury and illness in the backcountry compared with those without medical training, $(37.7 \%$ vs $20.7 \%, \mathrm{p}<.001)$. They were also more prepared for AMS $(62.3 \%$ vs $34.3 \%$, $\mathrm{p}<.001$ ). Respondents with wilderness training (WFA, WFR, and WEMT) were not significantly more likely to be prepared for illness and injury (31.8\% vs
$22.0 \%, \mathrm{p}<.11)$. However, they were more likely to be prepared for AMS $(52.3 \%$ vs $36.8 \%, \mathrm{p}=.025)$. Adjusting for experience, wilderness training, age, and gender, we found that medical training was associated with increased preparedness for injury and illness in the backcountry (2.71 Odds Ratio, 95\% CI 1.5-4.89) and AMS (2.72 Odds Ratio, 95\% CI 1.51-4.91).

Conclusions.-Medically trained and wilderness-trained individuals are more likely to be prepared for altitude and medical trauma than their nontrained counterparts. While it is neither practical nor feasible to train most hikers to be emergency medical technicians, nurses, or physicians, our data suggest that wilderness training is helpful in mitigating risk in the backcountry.

\section{Severe Hypertension and Efficacy of Antihyperten- sive Medications at High Altitude}

T. Douglas Sallade ${ }^{1}$; Linda E. Keyes ${ }^{2,3}$; Alison Sheets ${ }^{3}$; Jennifer Starling ${ }^{2,4}$; Sushil Pant ${ }^{5,6}$; David Young ${ }^{2}$; David Twillman ${ }^{2}$; Nirajan Regmi ${ }^{5}$; Benoit Phelan ${ }^{7}$; Purshotam Paudel ${ }^{5,8}$; Matthew McElwee ${ }^{9}$; Theodore McConnell ${ }^{10}$; Luke Mather ${ }^{11,12}$; Charles Duke ${ }^{13}$; Devlin Cole ${ }^{9}$; Buddha Basnyat ${ }^{14,15}$

${ }^{1}$ Philadelphia College of Osteopathic Medicine, Philadelphia, PA, USA, ${ }^{2}$ Department of Emergency Medicine University of Colorado, Aurora, CO, USA, ${ }^{3}$ Longmont United Hospital, Longmont, CO, USA, ${ }^{4}$ Colorado Permanente Medical Group, Saint Joseph Hospital, Denver, CO, USA, ${ }^{5}$ Mountain Medicine Society of Nepal, Kathmandu, Nepal, ${ }^{6}$ Khunde Hospital, Kathmandu, Nepal, ${ }^{7}$ Integrated Family MedicineEmergency Medicine Residency, Saint John Regional Hospital, Dalhousie University, Halifax, NS, Canada, ${ }^{8}$ District Hospital, Dhading, Nepal, ${ }^{9}$ Case Western Reserve University School of Medicine, Cleveland, OH, USA, ${ }^{10}$ McGill University, Montreal, QC, Canada, ${ }^{11}$ Family Medicine Residency of Idaho, Boise, ID, USA, ${ }^{12}$ University of Washington School of Medicine, Seattle, WA, USA, ${ }^{13}$ University of Tennessee Health Science Center, College of Medicine, Memphis, TN, USA, ${ }^{14}$ International Society of Mountain Medicine, Montreal, QC, Canada, ${ }^{15}$ Oxford University Clinical Research Unit-Nepal, Mountain Medicine Society of Nepal, Himalayan Rescue Association, Kathmandu, Nepal

Background.-Severe and uncontrolled hypertension have been reported in high altitude sojourners but have not been studied systematically. Additionally, few studies have evaluated the efficacy of antihypertensive medications at high altitude. We documented the prevalence of severe hypertension and efficacy of antihypertensive medications in high-altitude trekkers in Nepal.

Methods.-Observational cohort study in Nepal's Solukhumbu Valley. We recruited trekkers 18 years of age and older. Subjects reported demographics, medical history, and medications. Resting blood pressure (BP) was recorded at $2860 \mathrm{~m}, 3400 \mathrm{~m}$, and $4300 \mathrm{~m}$ on ascent and descent. Severe hypertension was defined as systolic BP $\geq 180$ and/or diastolic BP $\geq 100$.

Results.-We enrolled 60 self-reported hypertensive (HTN) trekkers and 606 normotensive (NTN) trekkers. Out of 2158 BP measurements at all altitudes, $109(5 \%)$ were severe. Of these, 39 occurred in $22(37 \%)$ HTN trekkers and 70 were distributed among $51(8 \%)$ NTN trekkers. Occurrence of severe hypertension was similar across altitudes $(P<.05)$. No subject was symptomatic. Among HTN trekkers, 10 took no antihypertensives and 5 of those had one or more severe BP measurements, accounting for $21 \%$ of all severe BPs in HTN trekkers. Seven out of 14 subjects on angiotensin converting enzyme inhibitors (ACEI) accounted for another one-third of all severe BPs. No subject taking a beta-blocker (BB), thiazide, ACEI + calcium channel blocker (CCB), ACEI + BB, or angiotensin receptor blocker (ARB) + alpha-1 antagonist had severe hypertension. Severe hypertension occurred in a smaller proportion of trekkers on combinations of $\mathrm{CCB}$ plus either ACEIs (0/3) or ARBs (1/3), and those taking more than 3 antihypertensives $(1 / 3)$

Conclusions.-Asymptomatic severe hypertension occurred at high altitude in normotensive and hypertensive subjects, but was more common in those with underlying hypertension. The clinical importance of these episodes is 